\title{
Possible association of idiopathic pulmonary hemosiderosis with rheumatoid arthritis: A case report
}

\author{
XIAOXIA REN ${ }^{1}$, TING YANG ${ }^{1}$, JIANMEI LI $^{2}$, JING ZHANG $^{3}$, JING GENG $^{1}$ and HUAPING DAI \\ ${ }^{1}$ Department of Pulmonary and Critical Care Medicine, Center of Respiratory Medicine, China-Japan Friendship Hospital; \\ ${ }^{2}$ National Clinical Research Center for Respiratory Diseases, Beijing 100029; ${ }^{3}$ Department of Pulmonary and \\ Critical Care Medicine, Kunming Municipal Hospital of Traditional Chinese Medicine, Kunming, \\ Yunnan 650051; ${ }^{4}$ Department of Pulmonary and Critical Care Medicine, Liaoning \\ Chaoyang Second Hospital, Chaoyang, Liaoning 122000, P.R. China
}

Received February 12, 2020; Accepted May 20, 2020

DOI: $10.3892 /$ etm.2020.8938

\begin{abstract}
Idiopathic pulmonary hemosiderosis (IPH) is a rare interstitial lung disease, usually occurring in children or young adults. Although several studies reported on the coexistence of IPH and immune system diseases, the association between these conditions has not been well described. The present study reports on the case of a 21-year-old female patient who presented with bilateral lung abnormalities. The patient was admitted due to a 2-year history of progressive exertional dyspnea, as well as arthralgia and joint swelling in the recent 2 months. During the past 15 years, the patient had been diagnosed with anemia and received repeated blood transfusions. Serial chest CT scans indicated an interstitial pattern. On physical examination, the patient had pale skin with a hemoglobin level of $65 \mathrm{~g} / 1$ and exhibited finger-clubbing. Arterial blood gas analysis revealed hypoxia. Anticyclic-citrullinated protein antibody and rheumatoid factor were highly positive. Pulmonary function tests revealed restrictive ventilation dysfunction and decreased diffusion capacity. Bronchoscopy and biopsy confirmed diffuse alveolar hemorrhage. Following assessment of the etiology, the diagnosis of IPH was made by
\end{abstract}

Correspondence to: Professor Huaping Dai or Professor Ting Yang, Department of Pulmonary and Critical Care Medicine, Center of Respiratory Medicine, China-Japan Friendship Hospital, 2 East Yinghua Road, Beijing 100029, P.R. China

E-mail: daihuaping@sina.com

E-mail: dryangting@qq.com

Abbreviations: $\mathrm{CCP}$, cyclic-citrullinated protein; $\mathrm{FEV}_{1}$, forced expiratory volume in 1 sec; FVC, forced vital capacity; HRCT, high-resolution CT; IDA, iron deficiency anemia; IPH, idiopathic pulmonary hemosiderosis; $\mathrm{PaO}_{2}$, arterial partial pressure of oxygen; RA, rheumatoid arthritis; RF, rheumatoid factor

Key words: idiopathic pulmonary hemosiderosis, interstitial lung disease, rheumatoid arthritis, immune system disease, case report exclusion. The patient's symptoms and laboratory findings combined also confirmed the diagnosis of rheumatoid arthritis (RA). After receiving corticosteroid treatment, the patient's condition improved, and she was discharged and followed up. Based on this patient and a review of the literature, the present study demonstrated for the first time that IPH may mediate the development of an RA pathology. Therefore, early diagnosis is important for the timely management of IPH, which may also delay or even prevent the development of immune system diseases, e.g. RA, in patients with IPH. Further attention should be paid to determine the association between IPH and immune system diseases in the clinical setting.

\section{Introduction}

Idiopathic pulmonary hemosiderosis (IPH) is a rare interstitial lung disease with an estimated incidence of 0.24 case/year/million in Swedish children and 1.23 cases/year/million in Japanese children $(1,2)$. IPH is characterized by diffuse parenchymal infiltrates, hemoptysis and secondary iron deficiency anemia (IDA) due to recurrent intrapulmonary capillary bleeding (3). Rheumatoid arthritis (RA) has at times been observed in patients with IPH $(4,5)$, which is of no known etiological significance. The present study reports on the case of a patient with IPH who also presented with RA and determined the possible association between IPH and RA, with the aim of helping physicians to identify such patients early and prevent illness and death.

\section{Case report}

A 21-year-old female patient was admitted to the China-Japan Friendship Hospital (Beijing, China) in March 2019 for confirmation of suspected IPH. From the age of 6 years, the patient had required repeated blood transfusions for anemia, and for the last 2 years, the patient had experienced exertional dyspnea, presenting with diffuse alveolar infiltration on chest CT. The patient also had occasional episodes of arthralgia and swelling of the wrist joints, elbows, shoulders, ankles, cervical spine and knees in the recent 2 months. In addition, the patient complained of coughing up sputum occasionally but denied 
hemoptysis. The patient reported a hospitalization 2 years previously due to conscious disturbance of unknown causes, which subsided after a short course of high-dose corticosteroids. However, the patient failed to recall any further details with regard to the etiology associated with her hospitalization. The patient was a non-smoker and reported no exposure to cytotoxic drugs, environmental or occupational allergens or toxic fumes, chemicals or dust.

On physical examination, the patient had pale skin. Auscultation of the lungs revealed mild end-inspiratory crackles in the bilateral lower lung zones. Cardiovascular, abdominal and neurological system examinations were unremarkable. Finger-clubbing was marked, but no skin lesions were noted.

The abnormal and most important clinically relevant laboratory test results were summarized in Table I. Arterial blood gas analysis revealed hypoxia. The hemoglobin level and hematocrit value were decreased. The platelet count, prothrombin time and activated partial thromboplastin time were normal. The reticulocyte count was elevated. A test for occult blood in the stools was negative. The direct and total serum bilirubin levels were normal. The serum iron protein level was within the normal limits. Hemoglobin electrophoresis, Coombs' test and test for cold agglutinins were negative. A bone marrow biopsy indicated iron deficiency. The erythrocyte sedimentation rate and $\mathrm{C}$-reactive protein were normal. Serum biochemistry, anticyclic-citrullinated protein (CCP) antibody (466 U/ml; upper limit, $<25 \mathrm{U} / \mathrm{ml}$ ) and rheumatoid factor (RF) $(1,710 \mathrm{IU} / \mathrm{ml}$; upper limit, <20 IU/ml) were highly increased and severely positive. Anti-nuclear antibody, anti-dsDNA, anti-Scl-70 antibody, complement C3 and C4, as well as anti-neutrophil cytoplasmic antibodies, were negative. A wide range of other laboratory parameters associated with infection was assessed and the results were negative or within normal limits.

The results of the pulmonary function tests are summarized in Table II and revealed restrictive ventilation dysfunction and decreased diffusion capacity; the forced vital capacity (FVC), forced expiratory volume in $1 \mathrm{sec}$ $\left(\mathrm{FEV}_{1}\right)$, total lung capacity and diffusion capacity of carbon monoxide were decreased. The ratio of $\mathrm{FEV}_{1}$ to $\mathrm{FVC}$ was also slightly decreased. Serial chest CT was performed using Aquilion 64-slice CT scanner (Toshiba Corp.) with intervals of $1.25 \mathrm{~mm}$ at the time of hospital admission. The scan was performed from the thoracic inlet to the lateral costophrenic angle, revealing an interstitial pattern with micronodules at the upper and middle zones of each lung lobe, as well as diffuse bilateral ground-glass opacities and patchy infiltrates at the lower lung lobes (Fig. 1A). The CT images were evaluated in consensus by 2 radiologists experienced in chest radiology for $>20$ years. Echocardiography did not reveal any significant valvular lesions. X-ray scans of the hands, elbows and knees were normal. Bronchoscopy was performed with a flexible bronchoscope (Olympus Corp.). The patient received topical anesthetic with oral mucosa (using lidocaine) and nasal passages (using oxybuprocaine gel). Bronchoalveolar lavage fluid was obtained from the posterior basal segment of the right lower lobe by administration followed by aspiration of $30 \mathrm{ml} 0.9 \% \mathrm{NaCl}$ and indicated the presence of hemosiderin-laden macrophages (51.5\%; Table III and Fig. 2A). There were five sequential instillations performed at the same site at 1-2-min intervals, and the volume used was five $30 \mathrm{ml}$ aliquots. Observation of progressively more hemorrhagic fluid with each successive lavage suggested alveolar hemorrhage (Fig. 2B).

Cryobiopsy samples were obtained from the left lower lobe by transbronchial lung cryobiopsy with a 1.9-mm probe (freezing time, $5-8 \mathrm{sec}$ ) (6). A total of 4 samples were taken. The frozen biopsy specimens were thawed in saline and placed in formalin to obtain tissue for histological analysis. Formalin-fixed lung tissues were paraffin-embedded and cut into 5- $\mu \mathrm{m}$ sections for staining with H\&E (Sigma-Aldrich; Merck KGaA), Masson trichrome and Perls (Prussian blue) staining (Nanjing Jiancheng Bioengineering Institute) according to the manufacturer's protocols and examined under a light microscope (Nikon Corp.). For confirmation of the histological diagnosis, all of the available lung biopsy specimens were reviewed by a pulmonary pathologist who was blinded to the patient's clinical and radiological information.

Histopathological examination revealed dense accumulations of hemosiderin-laden macrophages and erythrocytes invading into individual alveolar spaces, interalveolar septal thickening and fibrosis (Fig. 3). There was no evidence of concurrent vasculitis, granulomata or organizing alveolitis on light microscopy, which was considered to be consistent with the diagnosis of IPH $(7,8)$. Considering the results of the microscopic examination of the lung tissue and the broad panel of tests employed, which did not reveal the origin of the intra-alveolar bleeding, the diagnosis of primary IPH was established.

High positivity for anti-CCP [a member of the family of anti-citrullinated protein antibodies (ACPA)] was a significant characteristic of this patient. It is well-known that there is a close association between ACPA and bone destruction in RA (9). Previous studies support the significance of ACPA in inflammation-associated bone diseases, not confined to RA, but also in non-RA autoimmune conditions, and ACPA may be detected in psoriatic arthritis, systemic lupus erythematosus and juvenile idiopathic arthritis, as well as scleroderma and CREST syndrome, followed by Sjögren's syndrome and vasculitis (10-15). However, the prevalence of ACPA positivity was higher in patients with RA than in those mentioned above apart from RA $(11,12)$.

Furthermore, similar pulmonary infiltrates detected on the CT scan, as well as changes in pulmonary function, may be observed in patients with systemic sclerosis. However, the patient in the present study did not exhibit any typical clinical manifestations of systemic sclerosis, including skin thickening on the fingers, Raynaud's phenomenon and systemic sclerosis-associated autoantibodies.

Thus, in the patient of the present study, the combination of clinical presentation and laboratory results was consistent with a diagnosis of RA according to the American College of Rheumatology (ACR) criteria from 1987 (16) (arthritis of 11 joint areas, arthritis of hand joints, symmetric arthritis and serum RF) and the ACR/European League Against Rheumatism 2010 classification criteria (17) (joint swelling not better explained by any other disease, joint involvement of $>10$ major/intermediate/small joints and at least 2 small joints, highly positive RF and ACPA). In addition, the diagnosis of 
Table I. Relevant laboratory results of the reported case.

\begin{tabular}{|c|c|c|c|c|c|}
\hline \multirow[b]{2}{*}{ Parameter } & \multicolumn{4}{|c|}{ Result } & \multirow[b]{2}{*}{ Reference/negative range } \\
\hline & $03 / 21 / 2019$ & 05/05/2019 & 07/05/2019 & $11 / 25 / 2019$ & \\
\hline \multicolumn{6}{|l|}{ Hematology } \\
\hline Hemoglobin (g/l) & $56^{\mathrm{a}}$ & 119 & 132 & 138 & $115-150$ \\
\hline HCT (\%) & $21.2^{\mathrm{a}}$ & 41.1 & 41.6 & 40.9 & $35-45$ \\
\hline $\operatorname{MCV}(\mathrm{fl})$ & $65.8^{\mathrm{a}}$ & $73.3^{\mathrm{a}}$ & $80.3^{\mathrm{a}}$ & $80^{\mathrm{a}}$ & $82-100$ \\
\hline $\mathrm{MCH}$ (pg) & $17.4^{\mathrm{a}}$ & $21.2^{\mathrm{a}}$ & $25.5^{\mathrm{a}}$ & 27 & $27-34$ \\
\hline \multicolumn{6}{|c|}{ Immunology/serology } \\
\hline $\mathrm{CCP}(\mathrm{U} / \mathrm{ml})$ & $466^{\mathrm{a}}$ & & & $<25$ & $<25$ \\
\hline $\mathrm{RF}(\mathrm{IU} / \mathrm{ml})$ & $1710^{\mathrm{a}}$ & & & $77.5^{\mathrm{a}}$ & $<20$ \\
\hline \multicolumn{6}{|l|}{ Blood gas analysis } \\
\hline $\mathrm{PaO}_{2}(\mathrm{mmHg})$ & $66^{\mathrm{a}}$ & $76^{\mathrm{a}}$ & 85 & 85 & $83-108$ \\
\hline
\end{tabular}

${ }^{\mathrm{a} A b n o r m a l ~ r e s u l t . ~} \mathrm{HCT}$, hematocrit; $\mathrm{MCV}$, mean corpuscular volume; $\mathrm{MCH}$, mean corpuscular hemoglobin; CCP, anti-cyclic-citrullinated protein; $\mathrm{RF}$, rheumatoid factor; $\mathrm{PaO}_{2}$, arterial partial pressure of oxygen.

Table II. Results of pulmonary function tests.

\begin{tabular}{|c|c|c|c|c|c|}
\hline \multirow[b]{2}{*}{ Tests } & \multicolumn{2}{|c|}{ Result } & \multirow[b]{2}{*}{ Reference/negative range } & \multirow{2}{*}{$\frac{\text { Result }}{11 / 19 / 2019}$} & \multirow[b]{2}{*}{ Reference/negative range } \\
\hline & 03/20/2019 & 07/10/2019 & & & \\
\hline $\mathrm{FVC}(\mathrm{l})$ & $2.34^{\mathrm{a}}$ & $2.52^{\mathrm{a}}$ & 3.46 & $2.44^{\mathrm{a}}$ & 3.50 \\
\hline FVC\%pred (\%) & $67.60^{\mathrm{a}}$ & $72.90^{\mathrm{a}}$ & - & $69.60^{\mathrm{a}}$ & - \\
\hline $\mathrm{FEV}_{1}(\mathrm{l})$ & $1.76^{\mathrm{a}}$ & $1.76^{\mathrm{a}}$ & 3.02 & $1.80^{\mathrm{a}}$ & 3.06 \\
\hline $\mathrm{FEV}_{1} \%$ pred (\%) & $58.30^{\mathrm{a}}$ & $58.40^{\mathrm{a}}$ & - & $59.00^{\mathrm{a}}$ & - \\
\hline $\mathrm{FEV}_{1} / \mathrm{FVC}(\%)$ & $75.18^{\mathrm{a}}$ & $69.85^{\mathrm{a}}$ & - & $73.93^{\mathrm{a}}$ & - \\
\hline $\operatorname{TLC}(1)$ & $3.44^{\mathrm{a}}$ & 4.70 & 4.64 & $3.31^{\mathrm{a}}$ & 4.70 \\
\hline TLC \% pred (\%) & $74.20^{\mathrm{a}}$ & 101.30 & - & $70.30^{\mathrm{a}}$ & - \\
\hline DLCO $\%$ pred (\%) & $43.00^{\mathrm{a}}$ & $50.30^{\mathrm{a}}$ & - & $44.20^{\mathrm{a}}$ & - \\
\hline
\end{tabular}

${ }^{\mathrm{a}}$ Abnormal result. $\mathrm{FVC}$, forced vital capacity; $\mathrm{FEV}_{1}$, forced expiratory volume in 1 second; TLC, total lung volume; DLCO, diffusion capacity of carbon monoxide; \%pred, percentage of the predicted value.

RA was confirmed by an experienced rheumatologist. A final definitive diagnosis of RA and IPH was then established.

After the diagnosis of IPH and RA had been made, the patient began to receive high doses of oral prednisone $(0.75 \mathrm{mg} / \mathrm{kg})$ for 8 weeks, which was gradually tapered to $0.5 \mathrm{mg} / \mathrm{kg}$ for another 6 weeks. The treatment led to a profound improvement of dyspnea and arthralgia and a significant increase in hemoglobin levels, as well as a decrease of the CCP and RF titers (Table I). A control high-resolution CT (HRCT) obtained one month after steroid therapy revealed a marked regression of the aforementioned lesions observed on previous HRCT (Fig. 1B). Pulmonary function tests and arterial blood gas analysis were also performed, indicating an improvement during this period (Table II). The patient was followed up at 3 and 6 months after therapy by HRCT and no new lesions were observed (Fig. 1C and D). The patient has been closely monitored due to the high relapse incidence of IPH (18) and remained in remission at the time of the last follow-up in November 2019.
Table III. Results of bronchoalveolar lavage evaluation.

\begin{tabular}{lc}
\hline Cell type & Percentage \\
\hline Macrophages & 51.5 \\
Lymphocytes & 16.0 \\
Neutrophils & 31.5 \\
Eosinophils & 1.0 \\
\hline
\end{tabular}

\section{Discussion}

IPH is a rare form of interstitial lung disease characterized by recurrent intra-alveolar hemorrhage and secondary IDA, usually occurring in young adults or children (1). Various theories have been proposed to explain the etiology of $\mathrm{IPH}$, including genetic, environmental and auto-immune 


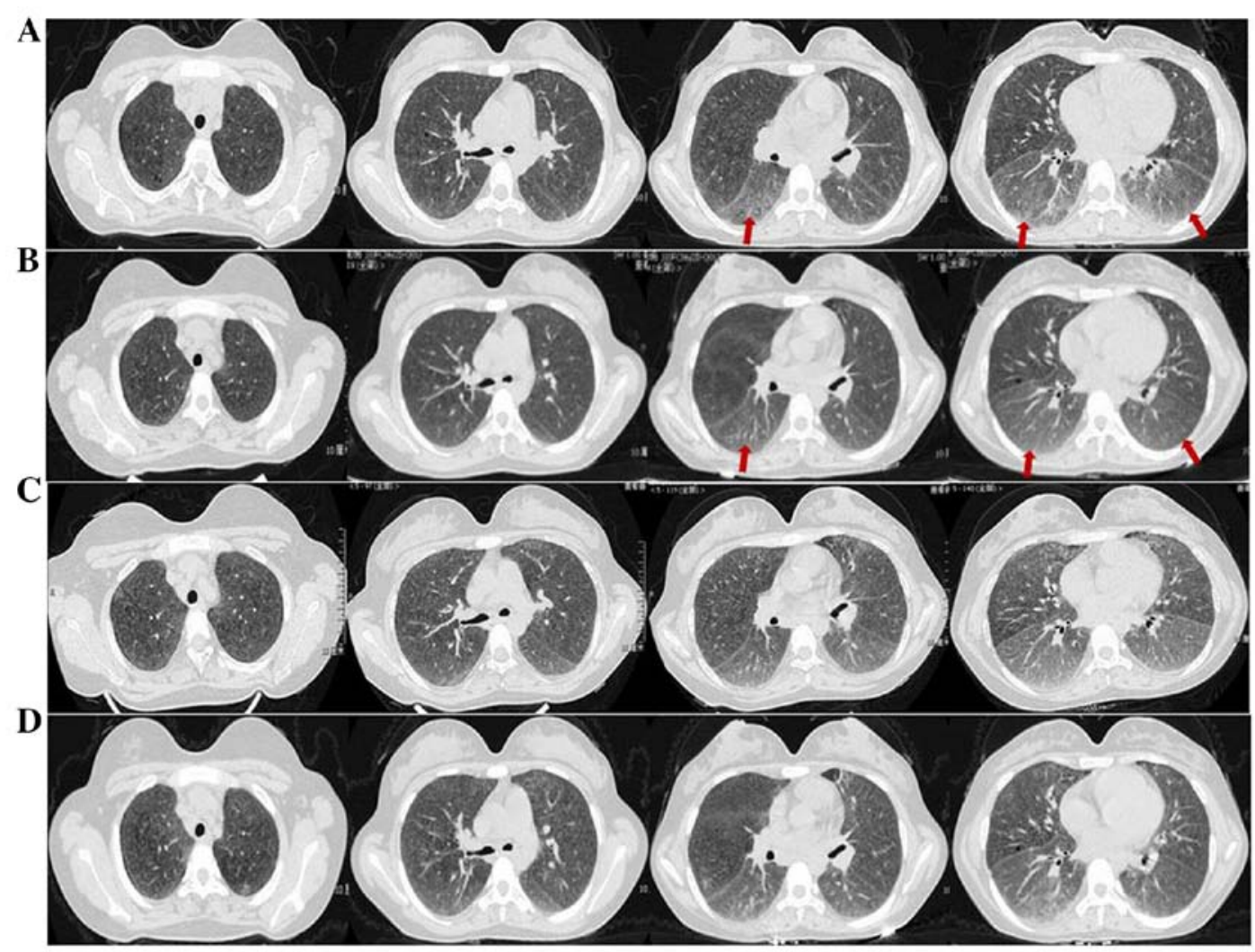

Figure 1. Axial CT was performed at the time of admission and follow-up at the hospital. (A) March 21, 2019: Diffuse interstitial pattern. (B) After 1 month: Marked regression of the aforementioned lesions (as indicated by the arrow). (C) After 3 months: No new lesions. (D) After 6 months: No new lesions.

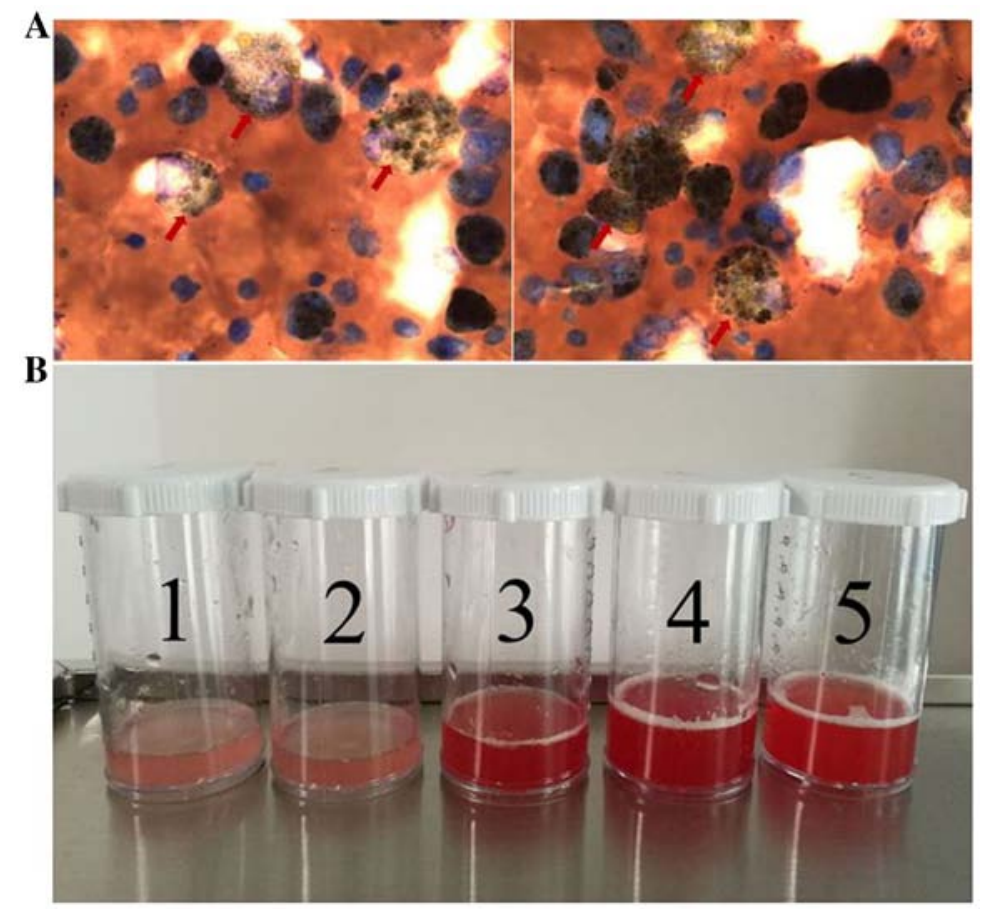

Figure 2. Flexible bronchoscopy with BAL was performed, and then processed for cell counts and differentials. (A) Numerous hemosiderin-laden macrophages (as indicated by the arrows) were observed in the BALF (hematoxylin \& eosin stain; magnification, x100). The left and right panel show different fields under a microscope. (B) Five sequential BALs were performed at the same site at 1-2-min intervals. The BALF appeared more hemorrhagic with successive lavage. BALF, bronchoalveolar lavage fluid.

mechanisms (19-24). The auto-immune theory provides the most acceptable explanation for the pathophysiology of
IPH, considering its frequent association with auto-immune diseases, including RA (24). 


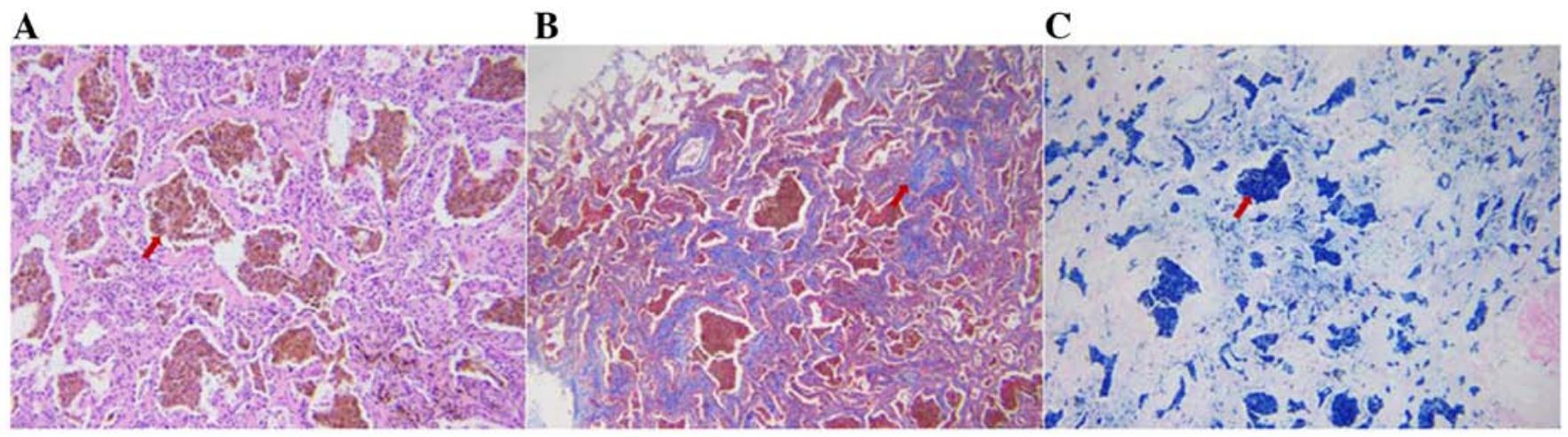

Figure 3. Transbronchial lung cryobiopsy was performed 3 days after admission. (A) Numerous hemosiderin-laden macrophages (as indicated by the arrow) were observed in the alveoli. The alveolar septum was thick and no vasculitis or capillaritis was observed (H\&E stain; magnification, $\mathrm{x} 100$ ). (B) There was a significant thickening of the collagen fibers in the alveolar septum (as indicated by the arrow) and hemosiderin-laden macrophages were observed in the alveoli (Masson stain; magnification, x100). (C) Numerous hemosiderin-laden macrophages (as indicated by the arrow) were observed in the alveoli and no vasculitis was observed (Perls stain; magnification, x100).

The coexistence of IPH and RA was first reported by Karlish (25), who described IPH concurring with early RA in a 32-year-old male patient. A study by Le Clainche et al (26) followed 15 pediatric patients with IPH, revealing that $25 \%$ of the patients who survive with the condition for $>10$ years subsequently develop autoimmune disorders, including RA. A total of 3 patients were reported to have developed RA at 6 months to 20 years after the initial diagnosis (26). In the patient of the present study, RA developed with a marked delay from the onset of symptoms (15 years). The subsequent development of RA is of considerable interest, since the association is insufficiently recognized due to the delayed occurrence of RA associated with IPH (26).

$\mathrm{RA}$ is the most frequent systemic disease in the general population (0.5-1.0\%) and arthritis is at times associated with respiratory symptoms, typically with diffuse parenchymal lung disease (27). Macrophages are central to the development of RA, and they may activate a wide range of immune cells and secrete diverse tissue-degrading enzymes mediating chronic pro-inflammatory, tissue-destructive and pain responses in RA (28). In the present case, the macrophages in the lung were abnormally activated (hemosiderin-laden macrophages), which is necessary for establishing the diagnosis of IPH by providing proof of chronic intra-alveolar bleeding (29). Furthermore, damage to pulmonary alveolar tissue by a variety of agents may induce a macrophage-centered autoimmune process in certain individuals, which may damage other non-pulmonary tissues, e.g. in arthrosis (30). Thus, RA may be caused by IPH through autoimmune responses, indicating IPH may precede the development of RA; a multicenter cohort, case-controlled study is required to confirm this.

Although IPH classically presents as a clinical triad, including IDA, episodes of recurrent hemoptysis and radiographic diffuse lung infiltrates, the clinical presentation varies greatly, from silent attacks to a fulminant course with rapidly worsening hypoxia $(2,8,23)$, which may cause a significant delay in the diagnosis of IPH. The quantity of hemoptysis may be variable and is not a reliable index of the degree of pulmonary hemorrhage, as alveolar bleeding does not readily reach the central airways (2). Hemoptysis is also minimal and goes unnoticed in children, as the sputum is mostly swallowed (31). Thus, hemoptysis is only detected in $50 \%$ of the patients (24), which leads to a delay between the onset of symptoms and the diagnosis of IPH. There is frequently a long delay of diagnosis, as the initial IDA usually precedes pulmonary signs for several months (32). In the patient of the present study, the delay in the diagnosis of IPH was by 15 years, since the onset of anemia was at the age of 6 years. This delay may result in pulmonary fibrosis due to iron overload within the lung, as free ferric ions may induce the formation of toxic hydroxyl radicals and/or stimulate fibrogenesis (33), which is generally a poor prognostic factor (34). The delay in diagnosis may be due to not only neglecting the cause of IDA, but also a lack of awareness of clinicians regarding the condition.

Systemic corticosteroids have been reported as the most effective treatment for IPH with favorable effects on alveolar bleeding relapse and pulmonary fibrosis progression, as well as with higher survival rates (35). Early treatment with steroids has also been widely indicated to shorten the duration of a hemorrhagic attack (36). Azathioprine in combination with corticosteroids was proposed to be the best therapeutic regimen, particularly for preventing IPH exacerbations (23). Considering the fertility of the case of the present study, the patient treated with prednisone only for a duration of 8 months and exhibited gradual improvement.

In conclusion, the current case provides supporting evidence regarding the IPH-mediated pathology of RA; thus, screening for autoimmune antibodies is recommended in patients with IPH. In addition, through the present case, the authors intend to emphasize that early IPH diagnosis and treatment are of grave importance for obtaining a favorable outcome, which may delay or even prevent the development of immune system diseases such as RA in patients with IPH.

\section{Acknowledgements}

The authors would like to thank Dr Ling Zhao (Department of Pathology, China-Japan Friendship Hospital, Beijing, China) and Professor Min Liu (Department of Radiology, China-Japan Friendship Hospital, Beijing, China) for their pathological and radiological advice on the case. 


\section{Funding}

This study was supported by the National Natural Science Foundation of China (grant no. 81800036) and the National Key Research and Development Program of China (grant no. 2016YFC0901102).

\section{Availability of data and materials}

All datasets used and/or analyzed during the current study are available from the corresponding author on reasonable request.

\section{Authors' contributions}

$\mathrm{XR}$ and TY analyzed and interpreted the patient data and wrote the manuscript. JL and JZ collected original medical records of the patient and drafted the manuscript. JG was in charge of the follow-up of the patient, and acquisition and analysis of these data. HD analyzed and interpreted the patient data and revised the manuscript. All authors read and approved the final manuscript.

\section{Ethics approval and consent to participate}

Approval was obtained from the Medical Ethics Committee of China-Japan Friendship Hospital (Beijing, China; no. 2018-148-K105). Written informed consent was obtained from the patient regarding participation in the study. The sample collection took place during hospitalization and the follow-up period.

\section{Patient consent for publication}

Informed consent was obtained from the patient regarding the publication of data and images.

\section{Competing interests}

The authors declare that they have no competing interests.

\section{References}

1. Kjellman B, Elinder G, Garwicz S and Svan H: Idiopathic pulmonary haemosiderosis in Swedish children. Acta Paediatr Scand 73: 584-588, 1984.

2. Ohga S, Takahashi K, Miyazaki S, Kato H and Ueda K: Idiopathic pulmonary haemosiderosis in Japan: 39 possible cases from a survey questionnaire. Eur J Pediatr 154: 994-998, 1995.

3. Al Jassmi AM: Autoimmunity and delayed diagnosis in pediatric idiopathic pulmonary hemosiderosis. J Pediatr Hematol Oncol 42: e240-e243, 2020.

4. Louie S, Russell LA and Richeson RB: Circulating immune complexes with pulmonary hemorrhage during pregnancy in idiopathic pulmonary hemosiderosis. Chest 104: 1907-1909, 1993.

5. Lemley DE and Katz P: Rheumatoid-like arthritis presenting as idiopathic pulmonary hemosiderosis: A report and review of the literature. J Rheumatol 13: 954-957, 1986.

6. Kania A, Misiaszek M, Vašáková M, Szlubowski A, Bugalho A, Pankowski J, Szołkowska M, Roden AC, Celejewska-Wójcik N, Nastałek P, et al: Cryobiopsy in the diagnosis of idiopathic pulmonary hemosiderosis: A case report. J Thorac Dis 11: 3195-3201, 2019.

7. Yao TC, Hung IJ, Jaing TH and Yang CP: Pitfalls in the diagnosis of idiopathic pulmonary haemosiderosis. Arch Dis Child 86 436-438, 2002

8. Ioachimescu OC, Sieber S and Kotch A: Idiopathic pulmonary haemosiderosis revisited. Eur Respir J 24: 162-170, 2004.
9. Hecht C, Englbrecht M, Rech J, Schmidt S, Araujo E, Engelke K, Finzel S and Schett G: Additive effect of anti-citrullinated protein antibodies and rheumatoid factor on bone erosions in patients with RA. Ann Rheum Dis 74: 2151-2156, 2015.

10. Saigal R, Bhakal SS, Goyal L and Goel AD: Seroprevalence of anti-citrullinated protein antibodies (ACPA) in patients with rheumatic diseases other than rheumatoid arthritis. J Assoc Physicians India 66: 26-28, 2018.

11. Yuan S, Chen D, Xiao Y, Lao M, Qiu Q, Liang L and Yang X: Factors associated with erosive arthritis in rheumatoid arthritis and other connective tissue diseases: A retrospective study from a southern Chinese population. J Clin Rheumatol 22: 22-29, 2016.

12. Conigliaro P, Chimenti MS, Triggianese P, Sunzini F, Novelli L, Perricone $\mathrm{C}$ and Perricone R: Autoantibodies in inflammatory arthritis. Autoimmun Rev 15: 673-683, 2016.

13. Pang SY, Liu HY, Huang YJ, Liu YF, Dai YM, Zeng P and Zeng HS: Diagnostic performance of anti-citrullinated protein/peptide antibodies in juvenile idiopathic arthritis. Genet Mol Res 15: gmr.15028641, 2016.

14. Wu R, Shovman O, Zhang Y, Gilburd B, Zandman-Goddard G and Shoenfeld Y: Increased prevalence of anti-third generation cyclic citrullinated peptide antibodies in patients with rheumatoid arthritis and CREST syndrome. Clin Rev Allergy Immunol 32: 47-56, 2007.

15. Cho SB, Lee JH, Ahn KJ, Bae BG, Kim T, Park YB, Lee SK, Lee KH and Bang D: Anti-cyclic citrullinated peptide antibodies and joint involvement in Behçet's disease. Yonsei Med J 53: 759-764, 2012.

16. Arnett FC, Edworthy SM, Bloch DA, McShane DJ, Fries JF, Cooper NS, Healey LA, Kaplan SR, Liang MH, Luthra HS, et al: The American Rheumatism Association 1987 revised criteria for the classification of rheumatoid arthritis. Arthritis Rheum 31: 315-324, 1988.

17. Aletaha D, Neogi T, Silman AJ, Funovits J, Felson DT, Bingham CO III, Birnbaum NS, Burmester GR, Bykerk VP, Cohen MD, et al: 2010 Rheumatoid arthritis classification criteria: An American College of Rheumatology/European League Against Rheumatism collaborative initiative. Arthritis Rheum 62: 2569-2581, 2010.

18. Zhang Y, Luo F, Wang N, Song Y and Tao Y: Clinical characteristics and prognosis of idiopathic pulmonary hemosiderosis in pediatric patients. J Int Med Res 47: 293-302, 2019.

19. Gencer M, Ceylan E, Bitiren M and Koc A: Two sisters with idiopathic pulmonary hemosiderosis. Can Respir J 14: 490-493, 2007.

20. Gerhardy B and Simpson G: Melioidosis and idiopathic pulmonary hemosiderosis: A cast-iron case. Respirol Case Rep 1: 46-47, 2013.

21. Cassimos CD, Chryssanthopoulos $\mathrm{C}$ and Panagiotidou C: Epidemiologic observations in idiopathic pulmonary hemosiderosis. J Pediatr 102: 698-702, 1983.

22. Nacaroglu HT, Sandal OS, Bag O, Erdem SB, Bekem Soylu O, Diniz G, Ozturk A and Can D: Association of celiac disease with idiopathic pulmonary hemosiderosis; Lane Hamilton Syndrome. Iran J Pediatr 25: e3312, 2015.

23. Chen XY, Sun JM and Huang XJ: Idiopathic pulmonary hemosiderosis in adults: review of cases reported in the latest 15 years. Clin Respir J 11: 677-681, 2017.

24. Taytard J, Nathan N, de Blic J, Fayon M, Epaud R, Deschildre A, Troussier F, Lubrano M, Chiron R, Reix P, et al: New insights into pediatric idiopathic pulmonary hemosiderosis: The French RespiRare $\left({ }^{\circledR}\right)$ cohort. Orphanet J Rare Dis 8: 161, 2013.

25. Karlish AJ: Idiopathic pulmonary haemosiderosis with unusual features. Proc R Soc Med 55: 223-225, 1962.

26. Le Clainche L, Le Bourgeois M, Fauroux B, Forenza N, Dommergues JP, Desbois JC, Bellon G, Derelle J, Dutau G, Marguet C, et al: Long-term outcome of idiopathic pulmonary hemosiderosis in children. Medicine (Baltimore) 79: 318-326, 2000.

27. Clement A, Nathan N, Epaud R, Fauroux B and Corvol H: Interstitial lung diseases in children. Orphanet J Rare Dis 5: 22, 2010.

28. Nagasawa Y, Takada T, Shimizu T, Narita J, Moriyama H, Terada M, Suzuki E and Gejyo F: Inflammatory cells in lung disease associated with rheumatoid arthritis. Intern Med 48: 1209-1217, 2009.

29. Cohen S: Idiopathic pulmonary hemosiderosis. Am J Med Sci 317: 67-74, 1999.

30. Smith BS: Idiopathic pulmonary haemosiderosis and rheumatoid arthritis. Br Med J 1: 1403-1404, 1966. 
31. Matsaniotis N, Karpouzas J, Apostolopoulou E and Messaritakis J: Idiopathic pulmonary haemosiderosis in children. Arch Dis Child 43: 307-309, 1968.

32. Kuhn MJ: Idiopathic pulmonary hemosiderosis: The importance of a chest radiograph in children with unexplained anemia. Mt Sinai J Med 52: 358-362, 1985.

33. Mateos F, Brock JH and Pérez-Arellano JL: Iron metabolism in the lower respiratory tract. Thorax 53: 594-600, 1998.

34. Casian A and Jayne D: Current modalities in the diagnosis of pulmonary vasculitis. Expert Opin Med Diagn 6: 499-516, 2012.
35. Bhatia S, Tullu MS, Vaideeswar P and Lahiri KR: Idiopathic pulmonary hemosiderosis: Alveoli are an answer to anemia. J Postgrad Med 57: 57-60, 2011.

36. Erkoçoğlu M, Civelek E and Kocabaş CN: Unusual presentation: Concurrent IgA deficiency and idiopathic pulmonary hemosiderosis. Pediatr Pulmonol 51: E34-E36, 2016. 